Research Article

\title{
Coverage Control Optimization Algorithm for Wireless Sensor Networks Based on Combinatorial Mathematics
}

\author{
Yongjie Wang (iD and Maolin Li \\ Yuncheng Vocational and Technical University, Yuncheng, Shanxi 044000, China \\ Correspondence should be addressed to Yongjie Wang; yjiewang67@outlook.com
}

Received 21 August 2021; Revised 23 September 2021; Accepted 19 October 2021; Published 18 November 2021

Academic Editor: Dragana S. Krstic

Copyright (C) 2021 Yongjie Wang and Maolin Li. This is an open access article distributed under the Creative Commons Attribution License, which permits unrestricted use, distribution, and reproduction in any medium, provided the original work is properly cited.

\begin{abstract}
The traditional wireless sensor network coverage control optimization algorithm has the problems of long completion time, high energy consumption, and low coverage. A new algorithm based on combinational mathematics for wireless sensor network coverage control is proposed. The particle swarm optimization (PSO) algorithm is used to optimize the coverage control process of wireless sensor networks. Then, the combined mathematics method is used to detect the local convergence problem. Finally, the quasi-physical forces of quasi-gravity and Coulomb force are used to integrate the quasi-physical force into the particle. In the process of velocity evolution, the speed correction process of particle swarm optimization is optimized, which can effectively avoid the local convergence problem of the particle swarm optimization algorithm, reduce the repeated coverage, and expand the coverage. The experimental results show that compared with the traditional algorithm, the proposed algorithm has short completion time, low energy consumption, and high coverage.
\end{abstract}

\section{Introduction}

Due to the large-scale and high-density deployment of wireless sensor network nodes in the target area, a large number of nodes overlap in the coverage area of the network. This overlap of coverage will lead to redundancy of data acquisition and transmission and channel interference, which wastes limited energy resources. On the premise of guaranteeing certain coverage, appropriate coverage control algorithm and node scheduling algorithm should be adopted to make the sensor modules of some nodes strategically dormant, which is of great significance to prolong the network lifetime.

At present, there are two strategies for the initial deployment of sensor networks. One is large-scale random deployment; the other is planned deployment for specific purposes. When the working environment of sensors is physically unreachable, nodes can only be deployed by random seeding, which is called random deployment [1]. On the contrary, when sensors can be accurately deployed to designated locations in the work area, it is called planned deployment. At present, this method is more common in practical applications and is suitable for scenarios where the number of network nodes is small $[2,3]$. Because sensor networks usually need large-scale, high-density deployment of nodes, most of them adopt random deployment. However, this large-scale random placement method is difficult to place a large number of sensor nodes in a suitable location at one time, and it is easy to cause the distribution of sensor nodes to be too dense or sparse, thus forming overlapping areas and blind areas of coverage. Therefore, after the initial deployment of sensor networks, coverage control strategy is needed to achieve ideal network coverage performance.

The coverage problem reflects the monitoring degree of sensor network nodes to designated areas, and it is a basic index to measure the quality of service in wireless sensor networks [4]. In sensor networks, different perception and communication ranges will have a greater impact on the deployment of nodes. The traditional multiobjective evolutionary algorithm studies the relationship between connectivity and coverage of sensor networks. For different application scenarios, researchers have proposed a large 
number of coverage mechanisms $[5,6]$. According to the mobility of nodes, coverage problems are divided into static coverage and mobile coverage.

At present, the coverage optimization method of particle swarm optimization [7] is easy to fall into local convergence. Therefore, a coverage optimization method for wireless sensor networks based on improved particle swarm optimization is proposed. The novelty of this method lies in integrating quasi-physical forces into the evolution process of particle velocity using quasi-gravity and Coulomb forces and optimizing the speed correction process of particle swarm optimization in order to achieve effective deployment of sensor nodes and maximize transmission. The overall framework of this method is as follows:

(1) Firstly, the concept and characteristics of wireless sensor networks are described, and the applications of wireless sensor networks in military, environmental monitoring, medical and health, smart home, industry, and so on, are explained. It illustrates the research significance of coverage control in wireless sensor networks.

(2) The particle swarm optimization (PSO) algorithm is used to optimize the coverage control process of wireless sensor networks.

(3) Combinatorial mathematics is used to detect local convergence. Two quasi-physical schemes, quasigravity and Coulomb force, are used to integrate quasi-physical forces into the particle velocity evolution process. The speed correction process of particle swarm optimization is optimized, which avoids the local convergence problem of particle swarm optimization, reduces the repetitive coverage, and optimizes the coverage area.

(4) Experiments verify the completion time, energy consumption, and coverage of different methods.

(5) The experimental results are further discussed.

(6) Conclusion: summarize the full text and look forward to the future.

\section{Related Works}

In the application of wireless sensor networks, coverage reflects the network's ability to monitor the physical world. It is often used as a standard to describe the quality of service (QoS) of wireless sensor networks and is the basis of the application of wireless sensor networks.

In recent years, there are many research works proposing theories and algorithms for the wireless sensor networks' coverage problem. Chakrabarty et al. [8] considered the grid coverage algorithms using integer linear programming to improve the surveillance area and localization accuracy. In addition, researchers also tried different coverage strategies, including Voronoi and Delaunay triangle, square, and hexagon $[9,10]$. At the same time, there are many challenges in using WSNs because of their properties. For example, each sensor needs to communicate with each other. Thus, they have a limited communication range and battery lifetime. The sensors need to be connected with each other to commute [11]. The coverage problem mainly focuses on monitoring a region of interest. Therefore, with a large number of sensors, they need a reasonable deployment for a better coverage. Meanwhile, the deployment algorithm should have an appropriate convergence rate.

To solve this problem, there are many heuristic algorithms proposed, including particle swarm optimization algorithm [12], simulated annealing algorithm [13], and ant colony optimization algorithm [14]. Particle swarm optimization algorithm (PSO) is a population-based stochastic search technique introduced by Kennedy and Eberhart in 1995 [15]. It is similar with the behavior of some animal groups, such as bird flocking. In this process, each bird can find food through social collaboration of neighboring birds, and the birds which have found food can guide other birds around them to fly to the food location. The sensors follow this social behavior to move to the own local optimum solution and the global optimum solution.

Based on the traditional particle swarm optimization algorithms, there are many works which proposed the novel algorithms to improve the performance. To improve the selfadaptive ability of sensors, $\mathrm{Wu}$ et al. [16] proposed an adaptive particle swarm optimization algorithm to add the degree of aggregation and evolution of the particle swarm to the inertia coefficient, which can reduce the overlap area in the search process. Liang and Suganthan [17] provided a dynamic multiswarm PSO by regrouping the swarms during the process to achieve a higher diversity. In addition, there are many research works about the distributed setting [18-20] to ensure a high parallelism and good performance. Zhao et al. [21] improved the dynamic multiswarm PSO by hybridizing it with harmony search and developed a new optimizer. In our work, we use combinatorial mathematics to discover the properties of the wireless sensor networks. Two quasi-physical schemes are integrated into the algorithm, which is different from the previous works. With these two quasi-physical forces, the convergence speed and coverage area can be improved.

In this paper, coverage is the main line, and coverage control technology of wireless sensor networks is studied. In combination with the practical application environment, coverage in wireless sensor networks is considered from a new perspective. Through the coverage control of wireless sensor networks, the deployment and energy saving of wireless sensor networks are studied in order to improve the reliability of deployment and the effectiveness of energy. It is of great significance to provide strong monitoring support for the practical application of wireless sensor networks.

\section{Algorithm Design}

In this section, we propose an algorithm for the wireless sensor networks' coverage problem. Our algorithm is based on the particle swarm algorithm [7]. However, it is prone to the local optimal solution problem in the iterative convergence process. Thus, we add the quasi-physical force algorithm to pursue the global optimal solution. Some adjustment and optimization methods are applied in our 
algorithm to improve the coverage efficiency, balance the distance of sensing nodes, and reduce overlapped coverage.

In the following part, we will introduce the particle swarm optimization algorithm in Section 3.1. To improve the coverage area and the convergence speed, we apply the quasi-physical force into it. In Section 3.2, the detailed definition and formula of the quasi-physical force are discussed. The improved particle swarm optimization algorithm integrated with the quasi-physical force is proposed in Section 3.3.

3.1. Particle Swarm Optimization Algorithm. Particle swarm optimization (PSO) is an optimization algorithm, in which each particle corresponds to a solution in the same solution region. Particles modify the current position and velocity according to the retrieval experience of their peers and their own. If there are particles in the particle swarm, particle $i(i=$ $1,2, \ldots, N)$ will modify its position and velocity according to the following equation:

$$
\left\{\begin{array}{l}
V_{i}=\omega * V_{i}+c_{1} * \operatorname{rand}()\left(p b e s t[i]-X_{i}\right)+c_{2} * \operatorname{rand}^{*}\left(p \operatorname{best}[g]-X_{i}\right), \\
X_{i}=X_{i}+V_{i},
\end{array}\right.
$$

where $x_{i}$ and $V_{i}$ are used to describe the position and velocity of the $i(i=1,2, \ldots, N)$ th particle; pbest $[i]$ is used to describe the past optimum position of particle $i$; pbest $[g]$ is used to describe the past optimum position of all particles; ran $d$ takes a random value in $[0,1]$; and $\omega$ is used to describe the inertia weight, which is the learning factor. This value is set to 1 .

Particle coding is to encode the position and velocity of the particle. If the points in the wireless sensor network include $X$ and $Y$ coordinates and the network coverage is used as the optimization target, the dimension space of the optimal location of the wireless sensor network node is $2 \mathrm{~N}$, and the particle encoding is also considered to be a vector with a capacity of $2 N$. The components within the vector are used to describe the position of the sensor node in the direction of $X$ or $Y$. The coding of each particle position is as follows:

$$
X=\left(X_{1}, Y_{1}, X_{2}, Y_{2}, \ldots, X_{n}, Y_{n}\right) .
$$

The particle velocities are coded as follows:

$$
V=\left(V_{1}^{X}, V_{11}^{Y}, V_{2}^{X}, V_{2}^{Y}, \ldots, V_{n}^{X}, V_{n}^{Y}\right) .
$$

The position and velocity codes of all particles in the particle swarm are consistent, and the position and velocity vectors of each particle are calculated independently.

To optimize the coverage of wireless sensor networks, the network coverage optimization objective functions and moving distance are comprehensively analyzed; that is, the fitness function is

$$
E=\max \left(f_{1} \operatorname{Cow}+f_{2} \frac{1}{D i s / N L+1}\right),
$$

where Cow is used to describe the coverage; Dis is used to describe the moving distance of the sensor node; $N$ is used to describe the number of sensor nodes in the target range; $L$ is used to describe the length of the rectangular area whose target range is $L \times L$; and $f_{1}$ and $f_{2}$ are used to describe the weights occupied by coverage and moving distance, respectively. Formula (4) comprehensively analyzes the changes in the coverage area of wireless sensor networks and the number of sensor nodes.

The flowchart of the particle swarm optimization algorithm is as follows:

(1) Initialization of the algorithm parameters and sensor node attributes.

(2) Initializing the particle swarm, in the target region, $2 N$ random numbers are used to form the initial position $(x, y)$ of the $N$ sensor nodes, and based on the vector composed of the positions, the position vectors of all the particles in the total particle group are initialized; then, the position vector of all particles in the particle group is $X=\left(X_{1}, Y_{1}, X_{2}, Y_{2}, \ldots, X_{n}, Y_{n}\right)$. Set the initial velocity of the particle to be 0 , and the velocity vector of all particles in the particle group is $V=\left(V_{1}^{X}, V_{11}^{Y}, V_{2}^{X}, V_{2}^{Y}, \ldots, V_{n}^{X}, V_{n}^{Y}\right)$.

(3) The fitness function of formula (4) is used to run the fitness value of particles.

(4) Iterative adjustment is performed on the velocity and position of the particle. If the algorithm performs the $k$ th iteration acquisition, the global optimal position $g$ best is replaced by the best position by pbest $[i], i=1,2, \ldots, N$, when all the particles search for the historical best position. When the algorithm performs the $k+1$ th iteration, the upper and lower limits of the velocity of the particles are set to be, respectively, $V_{\max }$ and $V_{\min }$, and the upper and lower limits of the position of the particles are pos ${ }_{\max }$ and pos $_{\text {min }}$, respectively.

(5) Cyclic iteration to analyze whether it meets the termination criteria. If it does, the algorithm ends and outputs the global optimum position; otherwise, the iteration cycle runs Step 3.

3.2. Quasi-Physical Force Algorithm. For the coverage problem of wireless sensor networks, it is important that the sensors are distributed evenly in the space, instead of gathering in parts of space. Thus, the quasi-physical force algorithm is to apply some force to keep the distance between sensors in an appropriate threshold. There are two schemes, quasi-universal gravitation and Coulomb force, which are proposed.

Quasi-gravitational model: the target range $A$ is equally divided into $m \times n$ pixels by a grid method. Each pixel is used as a mass point to constitute $m \times n$ mass points, and each sensor node is regarded as a circle whose perceptual radius is $r$. If the uncovered particle in the wireless sensor network is valuable to the adjacent circle, then the quasi-universal gravitational function of the $k$ th particle $v_{k}$ to the $i$ th circle $S_{i}$ based on the gravitational model is 


$$
f_{j k}= \begin{cases}\frac{r_{j}^{2}}{d_{j k}^{2}}, & v_{k} \notin \bigcup_{k=1}^{N} S_{n}, R_{j}>d_{j k}>r_{j}, \\ 0, & \text { else. }\end{cases}
$$

In the formula, $d_{j k}$ is used to describe the distance from a particle to the center of a circle. $r_{j}^{2}$ is used to describe the mass of a circle. $v_{k} \notin \cup \cup_{k=1}^{N} S_{n}$ is used to describe the gravitation of an uncovered particle to a circle; $R_{j}>d_{j k}>r_{j}$ restricts the region of action of gravity.

The Coulomb force model is constructed. Each sensor node acts as a Coulomb force between circles with a perceived radius of $r$. When the particle is not completely covered, there is no Coulomb force. When all the particles are covered, the existence of Coulomb force ensures that the sensor nodes in the circle are deployed in a balanced manner, reducing the problem of repeated coverage and obtaining the best coverage results. Set the Coulomb force function that is mutually exclusive between circles to be

$$
f_{i j}= \begin{cases}\frac{r_{i}^{2} r_{j}^{2}}{d_{i j}^{2}}, & d_{i j}<\min \left\{R_{i}, R_{j}\right\} \\ 0, & \text { else. }\end{cases}
$$

In the formula, $r_{i}^{2}$ and $r_{j}^{2}$ are the quantity of electricity by the circle; $d_{j k}$ is used to describe the distance between the centers of two circles; $d_{i j}<\min \left\{R_{i}, R_{j}\right\}$ can limit the area of action of the repulsive force between neighboring nodes.

Quasi-physical force algorithm can regulate the dispersion process of mobile sensor nodes. Quasi-gravitational force and Coulomb force can effectively regulate the distance of sensor nodes and reduce the repetitive coverage.

3.3. Improved Particle Swarm Optimization. A particle swarm optimization scheme guided by quasi-physical force is proposed by combining quasi-physical force algorithm and particle swarm optimization. The analysis of formula (1) shows that the evolution of particle velocity in particle swarm optimization is related to the optimal position of the particle and the optimal position of the particle swarm. The original position and velocity of the particle are generated randomly. If the number of particles is low, the optimization effect is poor, and some particles will produce regression deviating from the optimal solution. Therefore, in order to improve the convergence efficiency of particle swarm optimization and control the evolution of particles to the global optimal solution, the particle swarm optimization method guided by quasi-physical force integrates quasi-physical force into the evolution process of particle velocity, which can be described as

$$
\begin{aligned}
v_{i j}(n+1)= & W^{*} v_{i j}(n)+c_{1}^{*} \operatorname{rand}^{*}\left(p_{i j}-x_{i j}\right) \\
& +\operatorname{rand}^{*} c_{2}^{*}\left(p_{i j}-x_{i j}\right)+c_{3}^{*} \operatorname{rand}^{*} g_{1 i j}(n)+c_{4}^{*} \operatorname{rand}^{*} g_{2 i j}(n) .
\end{aligned}
$$

In the formula, $v_{i j}$ is the velocity of particle $i ; p_{i j}$ is the historical best position of particle $i ; c_{1}$ is the acceleration weight coefficient of the particle itself; $c_{2}$ is the global acceleration weight coefficient; $W$ is the inertia coefficient; $c_{3}$ and $c_{4}$ are the acceleration factors of quasi-gravitational force and Coulombic force, respectively; $g_{1 i j}(n)$ is the value force factor of quasi-gravitational force, which uses formula (5) to calculate the value of the gravitational function of a pixel for $j$ sensor nodes in $i$ particles. Formula (8) is used to calculate the sum value of the gravitational function of $K$ pixels:

$$
g_{1 i j}(n)=\sum_{k} f_{j k}=\sum_{k} \frac{r_{j}^{2}}{d_{j k}^{2}} .
$$

$g_{2 i j}(n)$ is the value force factor of Coulomb force. Formula (6) is used to calculate the Coulomb force function of one sensor node to other sensor nodes. Formula (9) is used to calculate the sum of the Coulomb force function of the sensor node to other nodes:

$$
g_{2 i j}(n)=\sum_{k} f_{i j}=\sum_{j} \frac{r_{i}^{2} r_{j}^{2}}{d_{i j}^{2}} .
$$

The running process of particle swarm optimization guided by quasi-physical force is as follows.

Integrating universal gravitation and Coulomb force into the particle swarm algorithm can reduce the repeated coverage problem of wireless sensor networks and improve the convergence efficiency. The specific process is as follows:

(1) Initialize the particle population number to arbitrarily form original solutions and original velocities, and calculate the original coverage of each particle

(2) Modify the velocity and position of each particle, and calculate the coverage of each particle's new position

(3) If the particle coverage is higher than the historical optimum value, the historical optimum value is set to the immediate fitness

(4) Retrieve the global optimum value based on the historical optimum solution of the particle

(5) Analyze whether the surrounding area is completely covered, and incorporate quasi-universal gravity and Coulomb force

(6) Repeatedly operate Steps (2) to (5) until the termination criteria are met

\section{Simulation}

In order to verify the comprehensive effectiveness of the proposed coverage control optimization algorithm for wireless sensor networks based on combinatorial mathematics, an experiment is needed. We implemented our algorithm using Python. We run the simulations on an Ubuntu 18.04.03 machine equipped with Intel(R) Core(TM) i7-8559U @2.70 GHz (4C8T) and 32 GB of RAM.

In this part, we will compare our improved particle swarm optimization algorithm (PSO) with other coverage control optimization algorithms. Owing to that our proposed improved particle swarm optimization algorithm performs better than the basic particle swarm optimization 
algorithm, we do not list their comparison in the simulation. There are two heuristic algorithms we mainly focus:

(1) Algorithm based on time synchronization [22] (TSA): in the algorithm, nodes exchange information with neighbors and work through the energy of running nodes. The other nodes make adjustments according to the distance and the working status.

(2) Genetic-based algorithm [23] (GA): it tries to optimize the coverage area and minimize the moving distance based on the initial locations of sensors. Each sensor can be regarded as a random generated individual. The fitness function is a combination of the coverage area and the moving distance. In each iteration, the new generation of solutions is determined to achieve a final result.

As the description mentioned above, we first compare their coverage area and the convergence speed. In Figure 1, it shows the coverage area with different induction radii of sensors. With the increased induction radius, the coverage area is increased. When the radius is large enough, the whole region can be covered. At the same time, we can see that our proposed algorithm performs better than the other two heuristic algorithms. The reason is that the quasi-physical force can reduce the overlapping area, and the sensors can collaborate with each other better.

The number of convergence iterations is shown in Figure 2. The number of iterations is inversely proportional to the induction radius. Actually, when the radius is large, the most area can be covered. In this way, the locations among all the sensors can be adjusted in a short time. While the radius is small, the moving speed is also slow and trying to find the optimal solution. In addition, we can also see that our proposed algorithm needs less number of iterations because the quasi-physical force mainly tries to solve the local optimum and the convergence issue.

In addition, we also compare our algorithm with the other two algorithms in the completion time and the energy consumption.

Analysis of Figure 3 shows that the coverage control optimization algorithm based on time synchronization for wireless sensor networks has the highest completion time, followed by the coverage control optimization algorithm based on genetic algorithm for wireless sensor networks. The proposed algorithm has a significantly shorter completion time than the coverage control optimization algorithm based on time synchronization for wireless sensor networks and the coverage control optimization algorithm based on genetic algorithm for wireless sensor networks. The network coverage control completion time of the optimization algorithm for coverage control of wireless sensor networks has higher network coverage control efficiency. The proposed algorithm is compared with the coverage control optimization algorithm based on time synchronization and the coverage control optimization algorithm based on genetic algorithm for wireless sensor networks in energy consumption of network coverage control. The experimental results are shown in Table 1.

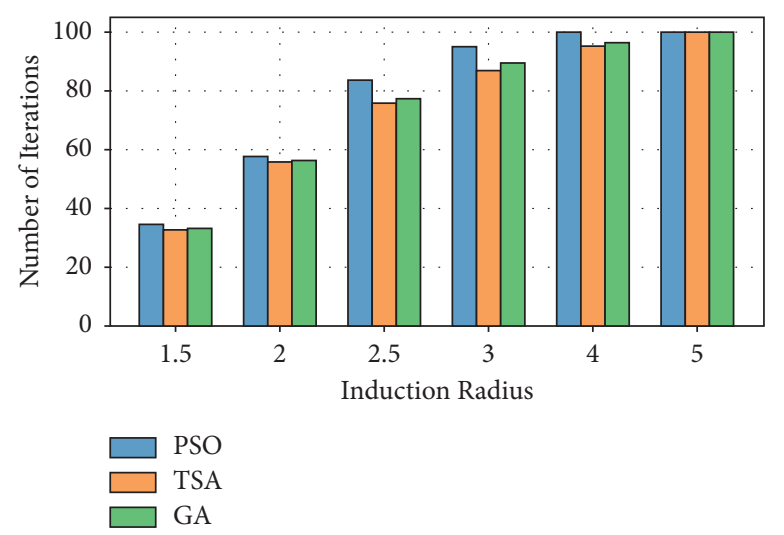

FIGURE 1: Rectangular graph of coverage varying with nodal induction radius.

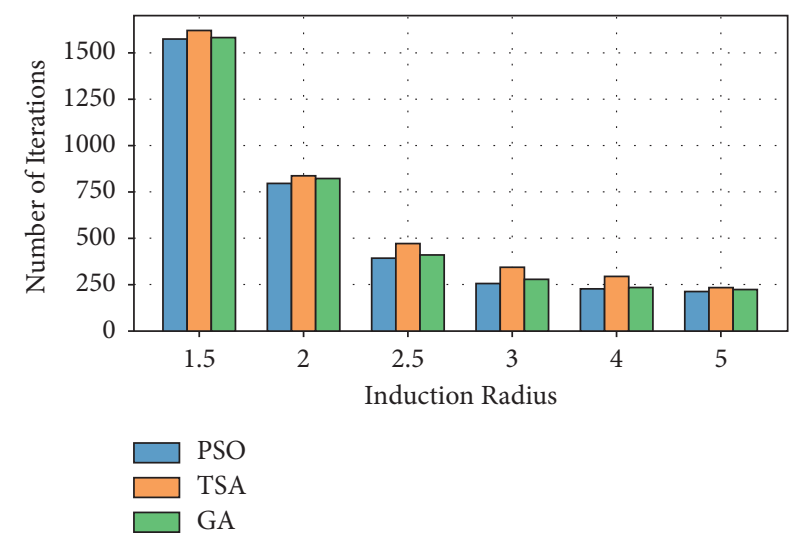

FIGURE 2: Rectangular graph with iteration number depending on node sensitive radius.

Analysis of Table 1 shows that the proposed algorithm has the lowest energy consumption of network coverage control among the three algorithms, followed by the coverage control optimization algorithm based on time synchronization for wireless sensor networks, and the coverage control optimization algorithm based on the genetic algorithm for wireless sensor networks has the highest energy consumption of network coverage control.

From the above simulation results, we have the following observations:

(1) Through Figures 1 and 2, the coverage rate of the proposed algorithm is higher, and the number of iterations is lower than that of the coverage control optimization algorithm based on time synchronization for wireless sensor networks, which shows that the proposed algorithm has better optimization performance and coverage performance.

(2) Comparing the completion time of different network coverage control algorithms, the proposed algorithm has the shortest completion time, followed by the genetic algorithm-based coverage control optimization algorithm for wireless sensor networks. The coverage control optimization algorithm based on 


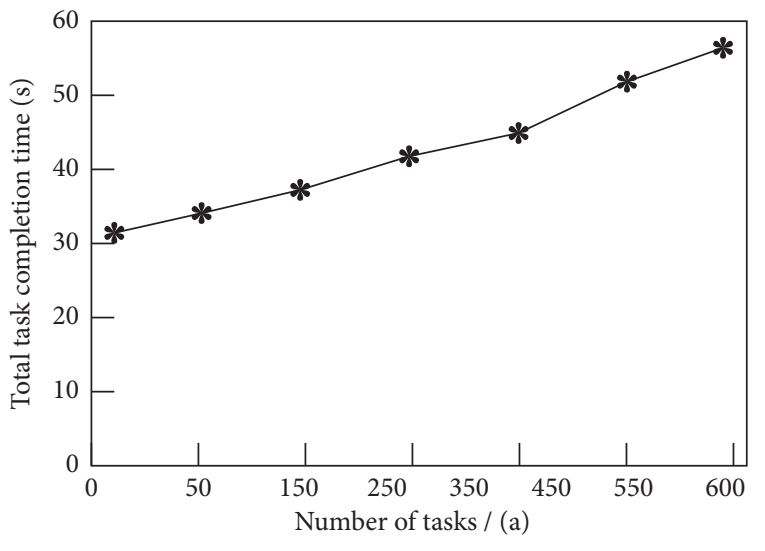

(a)

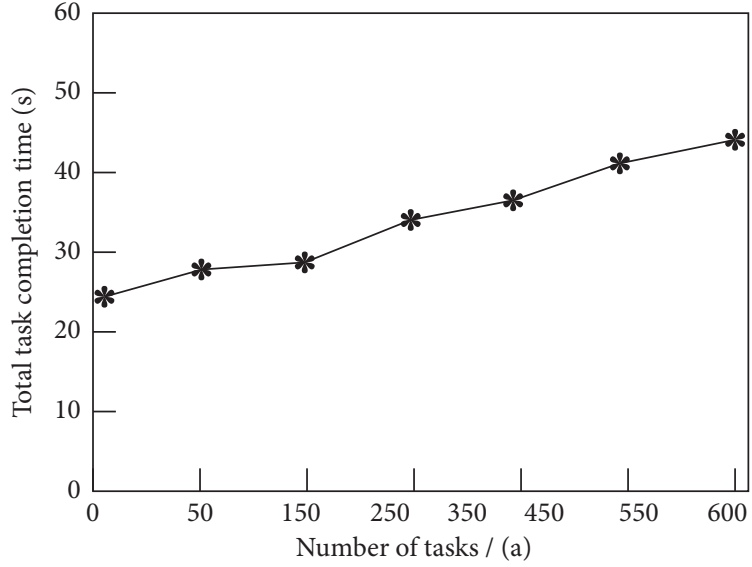

(b)

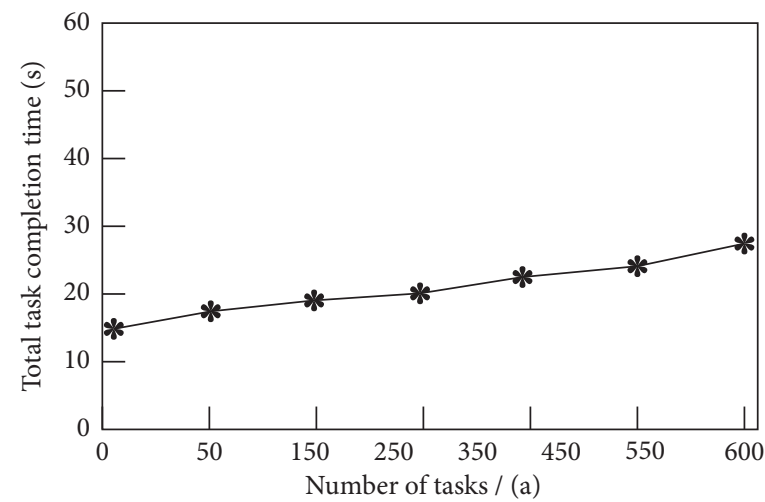

(c)

FIgURE 3: Comparison of completion time of network coverage control with different methods.

TABle 1: Comparison of energy consumption of network coverage control with different methods.

\begin{tabular}{llrr}
\hline Number of scheduled tasks (number) & \multicolumn{3}{c}{ Energy consumption (J) } \\
\hline 10 & PSO & TSA & 1354 \\
20 & 1102 & 1645 & 1954 \\
30 & 1354 & 1985 & 2132 \\
40 & 1411 & 2001 & 2415 \\
50 & 1547 & 2123 & 2879 \\
60 & 1699 & 2536 & 3321 \\
\hline
\end{tabular}

time synchronization for wireless sensor networks has the longest completion time, and the proposed algorithm has higher network coverage control efficiency.

(3) By comparing the energy consumption of different network coverage control algorithms, it can be seen that the energy consumption of the proposed algorithm is significantly lower than that of the other two algorithms, which has certain application performance.

\section{Conclusions}

Based on the particle swarm optimization (PSO) algorithm, this paper proposes a coverage control optimization algorithm-oriented quasi-physical force for wireless sensor networks. The quasi-physical force is used to improve the speed updating process of the PSO algorithm, guide the direction of particle evolution, accelerate the convergence of the algorithm, rationally adjust the distance of sensor nodes, and reduce repetitive coverage. The experimental results show that the proposed algorithm has shorter completion time, lower energy consumption, and higher coverage. The proposed method effectively solves the problems existing in traditional methods, lays a foundation for the progress of research in this field, reduces the waste of energy resources, and has great significance for prolonging network life. Outlook: due to time constraints, this paper only makes some attempts to improve the particle swarm optimization (PSO) algorithm in the study of coverage optimization in wireless sensor networks, but does not study other possibly 
better algorithms. If coverage optimization algorithm is to be applied to the real physical world, there are still many problems to be solved, which can be summarized as follows:

(1) Because sensor nodes are greatly limited in energy, computing power, and storage capacity, energysaving issues must be fully considered in the design so that the whole network can use as little energy as possible to complete the tasks that need to be completed.

(2) The current binary perception model and exponential perception model tend to be idealized. In the real physical world, the perception range of sensor nodes is irregular and changes with time. Therefore, the current perception model cannot well reflect the differences and time characteristics between sensor nodes, so more practical perception models should be used to design coverage control algorithm. We can consider introducing field theory to improve the model, which can make the model closer to the actual situation.

(3) When sensor nodes are used in battlefield or space exploration, coverage control in the three-dimensional space should be considered, which is still a complex problem.

(4) Currently, sensor nodes are generally considered to be incapable of mobility, but in most cases, sensor nodes are randomly distributed, and some areas monitored may be missing. In this case, we require the node to have the ability to move directly to the destination, so as to make up for the initial network coverage vulnerabilities.

\section{Data Availability}

All the data are generated by our simulation. It does not have any relation with other privacy and property issues.

\section{Conflicts of Interest}

The authors declare that they have no conflicts of interest.

\section{References}

[1] Y. D. Wang and P. Zhao, "Optimization of sensor network coverage based on improved pso algorithm," Systems Engineering and Electronics, vol. 39, no. 2, pp. 310-315, 2017.

[2] Y. Xu and Z. R. Wang, "Research on high-speed lte-r wireless network coverage and capacity optimization algorithm based on cooperative multi-agent mechanism," Journal of the Chinese Railway Society, vol. 39, no. 9, pp. 89-94, 2017.

[3] Y. Zhang and H. Y. Zhang, "Hybrid control of underwater acoustic sensor network deployment strategy based on virtual force and fruit fly optimization algorithm," Journal of Shanghai Jiaotong University, vol. 51, no. 6, pp. 715-721, 2017.

[4] Y. H. Li and X. Z. Duan, "Design and control performance analysis of interconnected grid load frequency controller based on intelligent optimization algorithm," Transactions of the Chinese Society of Electrical Engineering, vol. 33, no. 3, pp. 478-489, 2018.
[5] L. Xiao and X. H. Li, "Wireless video network routing communication protocol based on multi-angle optimization. control engineering," Control Engineering, vol. 24, no. 5, pp. 1038-1042, 2017.

[6] X. P. Hu and J. Cao, "Application of improved grey wolf optimization algorithm in wsn node deployment," Journal of Transducer Technology, vol. 31, no. 5, pp. 101-106, 2018.

[7] J. Kennedy and R. Eberhart, "Particle swarm optimization,"vol. 4, pp. 1942-1948, in Proceedings of the ICNN'95-international conference on neural networks, vol. 4, pp. 1942-1948, IEEE, Perth, Australia, November 1995.

[8] K. Chakrabarty, S. S. Iyengar, H. Hairong Qi, and E. Eungchun Cho, "Grid coverage for surveillance and target location in distributed sensor networks," IEEE Transactions on Computers, vol. 51, no. 12, pp. 1448-1453, 2002.

[9] E. S. Biagioni and G. Sasaki, "Wireless sensor placement for reliable and efficient data collection," in Proceedings of the IEEE, 36th Annual Hawaii International Conference on System Sciences, p. 10-, Burlingame, CA, USA, August 2003.

[10] K. Xu, G. Takahara, and H. Hassanein, "On the robustness of grid-based deployment in wireless sensor networks," in Proceedings of the 2006 International Conference on Wireless Communications and Mobile Computing, pp. 1183-1188, Bucharest, Romania, July 2006.

[11] N. A. B. Ab Aziz, A. W. Mohemmed, and B. D. Sagar, "Particle swarm optimization and voronoi diagram for wireless sensor networks coverage optimization," in Proceedings of the 2007 International Conference On Intelligent And Advanced Systems, pp. 961-965, IEEE, Kuala Lumpur, Malaysia, October 2007.

[12] Y. Yang, X. Fan, Y. Gan, Z. Zhuo, S. Wang, and P. Zhao, "Coverage optimization of sensor network based on improved particle swarm optimization," Systems Engineering and Electronics, vol. 39, no. 2, pp. 310-315, 2017.

[13] S. A. Feyzbakhsh, H. Saeedi, and S. F. Haqiqi, "A new approach to efficient sensor deployment on planar grid using the adam-eve genetic algorithm," GEM, pp. 197-205, 2007.

[14] D. Li, W. Liu, and L. Cui, "Easidesign: an improved ant colony algorithm for sensor deployment in real sensor network system," in Proceedings of the 2010 IEEE Global Telecommunications Conference GLOBECOM 2010, pp. 1-5, IEEE, Miami, FL, USA, December 2010.

[15] R. Eberhart and J. Kennedy, "Particle swarm optimization," Neural Networks, vol. 4, pp. 1942-1948, 1995.

[16] Y. Wu, Q. He, and T. Xu, "Application of improved adaptive particle swarm optimization algorithm in wsn coverage optimization," Chinese Journal of Sensors and Actuators, vol. 29, no. 4, pp. 559-565, 2016.

[17] J.-J. Liang and P. N. Suganthan, "Dynamic multi-swarm particle swarm optimizer," in Proceedings of the 2005 IEEE Swarm Intelligence Symposium, 2005, pp. 124-129, Pasadena, CA, USA, June 2005.

[18] S. Solomon, P. Thulasiraman, and R. Thulasiram, "Collaborative multi-swarm pso for task matching using graphics processing units," in Proceedings of the 13th Annual Conference on Genetic And Evolutionary Computation, pp. 1563-1570, New York, NY, USA, January 2011.

[19] X. Wang, J.-J. Ma, S. Wang, and D.-W. Bi, "Distributed particle swarm optimization and simulated annealing for energy-efficient coverage in wireless sensor networks," Sensors, vol. 7, no. 5, pp. 628-648, 2007.

[20] S. Su and S. Zhao, "A hierarchical hybrid of genetic algorithm and particle swarm optimization for distributed clustering in large-scale wireless sensor networks," Journal of 
Ambient Intelligence and Humanized Computing, vol. 9, pp. 1-11, 2017.

[21] S.-Z. Zhao, P. N. Suganthan, Q.-K. Pan, and M. Fatih Tasgetiren, "Dynamic multi-swarm particle swarm optimizer with harmony search," Expert Systems with Applications, vol. 38, no. 4, pp. 3735-3742, 2011.

[22] G.-Q. Chang, X.-P. Fan, and S.-Q. Liu, "Coverage control optimization algorithm in wireless sensor networks on time synchronization," Application Research of Computers, vol. 1, 2012.

[23] N. Rahmani, F. Nematy, A. M. Rahmani, and M. Hosseinzadeh, "Node placement for maximum coverage based on voronoi diagram using genetic algorithm in wireless sensor networks," Australian Journal of Basic and Applied Sciences, vol. 5, no. 12, pp. 3221-3232, 2011. 